\title{
EDITORIAL
}

\section{Drug Discovery for Disorders of the Central Nervous System}

Never in the history of science and medicine have we had a better understanding of brain function and the diseases that lead to its ultimate destruction and dysfunction. We have teased apart much detail concerning pathological cascades, genetic mutations, and environmental influences leading to some of the most devastating illnesses afflicting mankind in the post-vaccination world. Nevertheless, the majority of neurotherapeutics developed over the past three decades have been derived from serendipitous observations or oversimplified neurochemical hypotheses; the dopaminergic dysfunction of Parkinson's disease, the acetylcholine deficit observed in the basal forebrain of Alzheimer's disease patients, and the dopaminergic hyperfunction of schizophrenia are three such examples. Such hypotheses have led to the development and commercialization of medicines that, although providing clear benefit to patients, have still left much room for improvement in terms of both efficacy and tolerability. These therapies also help to highlight many of the limitations of our disease understanding because they are fairly uniformly unable to address the underlying etiology of any of the devastating diseases they are aimed to treat. Moreover, despite the increasing knowledge of the underlying substrates of neurological and psychiatric disease, despite dramatic increases in our ability to use biomarkers and imaging to mechanistically assess the potential actions of new therapeutics, and despite massive industrial investments in the development of new therapies for CNS diseases, success rates for pharmaceutical development have reached historical lows over the past several years. In this issue of NeuroRx ${ }^{\circledR}$, better understanding of the characteristics of successful therapeutics (Pajouhesh and Lenz) and better strategies to adapt to emerging legal processes and standards needed to protect intellectual property (Gersten) are both seen as needed to improve the possibility for successful commercial development of new therapies.

The era of the human genome has now revealed a bountiful supply of innovative and exciting drug discovery targets filled with potential for treating not only the symptoms but perhaps even the underlying causes of seemingly incurable neurological and neuropsychiatric diseases. Furthermore, the advent of exponential im-

Address correspondence and reprint requests to Menelas N. Pangalos, Ph.D., Vice President Neuroscience Research, Wyeth Research, CN8000, Princeton, NJ 08543. provements in areas such as high-throughput screening, robotics, molecular modeling, and data handling, coupled with the large advances in medicinal chemistry capacity and output, have meant that the number of targets now available for drug discovery efforts is at an all-time peak. Although the hopes and expectations of the world for breakthrough therapies may be high, never before have the challenges facing drug discovery scientists been harder. In fact, it is clear that the more we discover and understand about diseases of the CNS, the more surprises we find and the more we realize that there is much we do not understand. Whereas clinical diagnostics have focused on common phenotypes, deeper investigation reveals that the underlying causes of common phenotypes can be diverse and not susceptible to a single treatment intervention. Even the most seemingly simple genetic disorder, cystic fibrosis for example, can be caused by as many as 1000 different gene mutations, each one resulting in a subtly different biochemical change. One mutation may alter the maximum velocity of a protein, another the Michaelis constant, and a third may produce a functionally normal protein with a different stability or a tendency to aggregate. The chance a single intervention would reverse these three different defects causing a common phenotype is small. Understanding that diseases are not homogeneous in a patient population is perhaps one of the most significant realizations of modern medicine and drug discovery, and yet this knowledge now opens the next immense set of challenges for the field of neurotherapeutics. Using the collective tools of translational medicine such as gene expression profiling, biomarker identification, pharmacogenomics, and neuroimaging, we will be in a better position to diagnose and characterize the pathophysiological state of patient's disease, resulting in therapies better tailored to the individuals unique needs. In this issue, new approaches in understanding the nature of disease and the process of drug action (Hilbush et al., Gomez-Mancilla and colleagues, and Hurko and Ryan) promise to improve the choice of therapeutic targets and the preclinical validation of therapeutic agent action as well as improve patient selection and so enhance the eventual probability of success of materials placed into clinical trials. Even if such therapies are still years away, it is clear that our best chance of delivering on these aspirations is through the combined collaborative efforts of academic, government, regulatory, and industrial partnerships around the globe. 
In the pages to follow, we have put together an issue demonstrating examples of the strategies being pursued, ranging from the very earliest stages of target identification through to the discovery and validation of efficacy biomarkers in the clinic. The richness and complex interaction of systems underlying CNS function and pathophysiology generate enormous intellectual challenges for our community of neurotherapeutic developers. In this issue, some of the CNS disease targets where increased understanding has opened up opportunities for transformative therapies (Ossipov and Porecca, Snutch, Virley, Pangalos and colleagues, Schechter et al., and Marek and Merchant) are described. The hurdles for drug discovery scientists remain enormous, but as we increase our un- derstanding of brain function and pathophysiology we will surely better address the challenge of successfully developing drugs that can change the treatment paradigm for patients. Ultimately, our success over the coming decade will be determined by future therapies reaching the marketplace and whether we are able to move away from palliative symptomatic therapies and on to the "holy grail" of disease-modifying treatments.

Menelas N. Pangalos, Ph.D. E-mail: pangalm@wyeth.com Christopher C. Gallen, M.D., Ph.D. E-mail: cgallen@neuromed.com Guest Editors 